\title{
Tree Phyllosphere Bacterial Communities: exploring the magnitude of intra- and inter-individual variation among host species
}

\author{
Isabelle Laforest-Lapointe ${ }^{\text {Corresp., }}{ }^{1,2}$, Christian Messier ${ }^{1,2,3}$, Steven W. Kembel ${ }^{2}$ \\ ${ }^{1}$ Centre d'étude de la forêt, Montreal, Canada \\ 2 Sciences Biologiques, Université du Québec à Montréal, Montreal, Quebec, Canada \\ 3 ISFORT - Institut des Sciences de la Forêt Tempérée, Université du Québec en Outaouais, Ripon, Quebec, Canada \\ Corresponding Author: Isabelle Laforest-Lapointe \\ Email address: isabelle.laforest.lapointe@gmail.com
}

Background. The diversity and composition of the microbial community of tree leaves (the phyllosphere) varies among trees and host species and along spatial, temporal, and environmental gradients. Phyllosphere community variation within the canopy of an individual tree exists but the importance of this variation relative to among-tree and among-species variation is poorly understood. Sampling techniques employed for phyllosphere studies include picking leaves from one canopy location to mixing randomly selected leaves from throughout the canopy. In this context, our goal was to characterize the relative importance of intra-individual variation in phyllosphere communities across multiple species, and compare this variation to inter-individual and interspecific variation of phyllosphere epiphytic bacterial communities in a natural temperate forest in Quebec, Canada.

Methods. We targeted five dominant temperate forest tree species including angiosperms and gymnosperms: Acer saccharum, Acer rubrum, Betula papyrifera, Abies balsamea and Picea glauca. For one randomly selected tree of each species, we sampled microbial communities at six distinct canopy locations: bottom-canopy (1-2m height), the four cardinal points of mid-canopy (2-4m height), and the top-canopy (4-6m height). We also collected bottom-canopy leaves from five additional trees from each species.

Results. Based on analysis of bacterial community structure measured via Illumina sequencing of the bacterial $16 \mathrm{~S}$ gene, we demonstrate that $65 \%$ of the intra-individual variation in leaf bacterial community structure could be attributed to the effect of inter-individual and inter-specific differences while the effect of canopy location was not significant. In comparison, host species identity explains $47 \%$ of interindividual and inter-specific variation in leaf bacterial community structure followed by individual identity (32\%) and canopy location (6\%).

Discussion. Our results suggest that individual samples from consistent positions within the tree canopy from multiple individuals per species can be used to accurately quantify variation in phyllosphere bacterial community structure. However, the considerable amount of intra-individual variation within a tree canopy ask for a better understanding of how changes in leaf characteristics and local abiotic conditions drive spatial variation in the phyllosphere microbiome. 
1 TYPE OF ARTICLE: Original Article

2

3

4 TITLE: Tree Phyllosphere Bacterial Communities: exploring the magnitude of intra- and inter-

5 individual variation among host species

10 Isabelle Laforest-Lapointe ${ }^{1,2 *}$, Christian Messier ${ }^{1,2,3}$ and Steven W. Kembel ${ }^{1,2}$

11 Département des sciences biologiques, Université du Québec à Montréal, Montréal, (H3C

12 3P8), Québec, Canada

$13{ }^{2}$ Centre d'étude de la forêt, Université du Québec à Montréal, Montréal, (H2X 3Y7), Québec,

14 Canada

$15{ }^{3}$ Institut des Sciences de la Forêt tempérée, Université du Québec en Outaouais, Ripon, (J0V

16 1V0), Québec, Canada

17

18 * Correspondence: Isabelle Laforest-L., Département de Sciences Biologiques, Université du

19 Québec à Montréal, 141 av. Président-Kennedy, Montréal, (H2X 3Y7), Québec, Canada. Phone:

20 514-987-3000 (6936). Email: isabelle.laforest.lapointe@gmail.com 


\section{ABSTRACT}

23

24 Background. The diversity and composition of the microbial community of tree leaves (the

25 phyllosphere) varies among trees and host species and along spatial, temporal, and

environmental gradients. Phyllosphere community variation within the canopy of an individual

tree exists but the importance of this variation relative to among-tree and among-species

variation is poorly understood. Sampling techniques employed for phyllosphere studies include

picking leaves from one canopy location to mixing randomly selected leaves from throughout

the canopy. In this context, our goal was to characterize the relative importance of intra-

individual variation in phyllosphere communities across multiple species, and compare this

variation to inter-individual and interspecific variation of phyllosphere epiphytic bacterial communities in a natural temperate forest in Quebec, Canada.

Methods. We targeted five dominant temperate forest tree species including angiosperms and gymnosperms: Acer saccharum, Acer rubrum, Betula papyrifera, Abies balsamea and Picea glauca. For one randomly selected tree of each species, we sampled microbial communities at six distinct canopy locations: bottom-canopy (1-2m height), the four cardinal points of midcanopy (2-4m height), and the top-canopy (4-6m height). We also collected bottom-canopy leaves from five additional trees from each species.

42 Results. Based on analysis of bacterial community structure measured via Illumina sequencing of the bacterial $16 \mathrm{~S}$ gene, we demonstrate that $65 \%$ of the intra-individual variation in leaf bacterial community structure could be attributed to the effect of inter-individual and inter- 
45 specific differences while the effect of canopy location was not significant. In comparison, host

46 species identity explains $47 \%$ of inter-individual and inter-specific variation in leaf bacterial

47 community structure followed by individual identity (32\%) and canopy location $(6 \%)$.

49 Discussion. Our results suggest that individual samples from consistent positions within the tree

50 canopy from multiple individuals per species can be used to accurately quantify variation in

51 phyllosphere bacterial community structure. However, the considerable amount of intra-

52 individual variation within a tree canopy ask for a better understanding of how changes in leaf

53 characteristics and local abiotic conditions drive spatial variation in the phyllosphere

54 microbiome. 
55

56

57

58

\section{INTRODUCTION}

The phyllosphere microbiota represents the communities of microorganisms including bacteria, archaea, and eukaryotes such as fungi that are associated with plant leaves (Inácio et al. 2002; Lindow \& Brandl 2003). Phyllosphere microbes influence host fitness through a variety of mechanisms such as plant hormone production and protection from pathogen colonization (Innerebner et al. 2011; Ritpitakphong et al. 2016). As a result of their effect on host plant fitness, leaf microorganisms can influence plant population dynamics and community diversity (Clay \& Holah 1999; Bradley et al. 2008) as well as ecosystem functions including water (Rodriguez et al. 2009) and nutrient cycling (van der Heijden et al. 2008; McGuire \& Treseder 2010; Allison \& Treseder 2011). Tree microbial phyllosphere communities have been studied in tropical (Lambais et al. 2006, 2014; Kim et al. 2012; Kembel et al. 2014; Kembel \& Müller 2014), temperate (Jumpponen \& Jones 2009; Redford \& Fierer 2009; Redford et al. 2010; Jackson \& Denney 2011) and Mediterranean forests (Penuelas et al. 2012), along altitudinal gradients (Cordier et al. 2012ab), and in deserts (Finkel et al. 2011, 2012). In order to understand the structure and function of phyllosphere microbial communities, studies typically either assume that a single sample of leaves from a plant canopy is representative of the phyllosphere community of the entire tree or host species (Lambais et al. 2006; Kim et al. 2012; Kembel et al. 2014), or control for spatial structure in phyllosphere community structure by mixing leaves from multiple canopy locations (Redford \& Fierer 2009; Redford et al. 2010; Jumpponen \& Jones 2009, 2010; Finkel et al. 2011, 2012; Cordier et al. 2012ab). In this study our aim was to quantify the relative importance of intra-individual versus inter-individual and inter-specific variation in the structure of temperate tree phyllosphere communities across multiple host species. 

identity (Redford et al. 2010; Kembel et al. 2014) are important drivers of phyllosphere bacterial community structure. Most studies of phyllosphere communities across different host species

81 have assumed within-plant and within-species variation in phyllosphere community structure to

82 be negligible, and looked passed intra-individual and inter-individual variation (but see Redford et al. 2010 and Leff et al. 2015). In tree phyllosphere studies, samples are usually taken from

84 shade leaves either at the bottom of the canopy or at mid-canopy height near the trunk. However, 85 the technique to sample phyllosphere communities vary between studies, ranging from studies 86 that sampled leaves from a specific canopy location (i.e. Kembel et al. 2014; Kembel \& Müller 2014) to taking multiple leaves from around the canopy at the same height (i.e. Redford \& Fierer 2009; Redford et al. 2010; Jackson \& Denney 2011). However, Leff et al. 2015 demonstrated for a single tree species (Ginkgo biloba) that there is intra-individual variation in phyllosphere community structure within the canopy of a single tree. The relative importance of this withinindividual variation versus inter-individual and inter-specific variation, and the degree to which a sample of leaves from a canopy are representative of the microbiome of an individual or a species, is not well understood.

A multitude of factors could influence microbial community structure on leaves within a tree canopy. Leaf position in the canopy defines the degree of exposure to ultraviolet radiation

97 and wind and therefore community structure could change depending on the position of the

98 leaves sampled. Exposure to ultraviolet radiation has been shown to increase the diversity of the

99 maize leaf microbial community (Kadivar \& Sapleton 2003) and anoxygenic phototropic

100 bacteria have been detected in the phyllosphere of Tamarix nilotica (Atamna-Ismaeel et al. 
101 2012). This phenomenon could also be caused by leaf morphological and ecophysiological

102 attributes associated with high light availability (thicker leaves, lower specific leaf area, lower

103 water content, higher total chlorophyll, higher photosynthetic activity rate; Lichtenthaler et al.

104 2007). Variation in atmosphere conditions within the canopy (i.e. increased exposure to wind and

105 gas exchange levels) modifies local leaf humidity conditions potentially influencing leaf

106 epiphytic bacterial communities by inhibiting or favoring the growth of particular groups

107 (Medina-Martìnez et. al 2015). Wind exposure could reduce leaf moisture and induce a stomata

108 closure (Grace et al. 1975), which could impact the diffusion of nutrients and reduce the size of

109 microbial aggregates (Leveau \& Lindow 2001; Miller et al. 2001).

111 In this study, we aim to (1) compare the intra-individual, inter-individual and

112 interspecific variation of phyllosphere bacterial communities; (2) characterize the composition of

113 epiphytic phyllosphere bacterial communities at different canopy locations for five tree species;

114 and (3) make practical recommendations for the sampling of tree phyllosphere bacterial

115 communities. We hypothesized that (1) the magnitude of intra-individual variation will be

116 smaller than inter-individual and interspecific variation, (2) that canopy location will be a

117 significant driver of phyllosphere bacterial community structure because of variation in abiotic

118 conditions (e.g. radiation, wind), and changes in ecophysiological and morphological leaf

119 characteristics.

120

121 MATERIALS AND METHODS

122 Study Site \& Host-Tree Species 
123 The two study sites are located in a natural temperate forest stand in Gatineau $\left(45^{\circ} 44^{\prime} 50^{\prime \prime} \mathrm{N}\right.$;

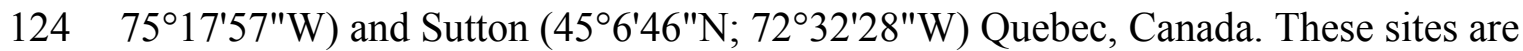

125 characterized by a cold and humid continental climate with temperate summer. A total of six

126 individuals (three at each site) from each of five tree species common to temperate forests and

127 dominant in the canopy were sampled to provide representatives of both angiosperms and

128 gymnosperms: Abies balsamea (Balsam fir), Acer rubrum (Red maple), Acer saccharum (Sugar

129 maple), Betula papyrifera (Paper birch) and Picea glauca (White spruce).

\section{Bacterial community collection}

132 We sampled phyllosphere communities from trees on August 29, 2013 as part of another

133 experiment (Laforest-Lapointe et al. 2016). Sampling was carried out one week after the last

134 rainfall event. We defined three strata within the canopy: bottom-canopy (1-2m height), mid-

135 canopy (2-4m height), and top-canopy (4-6m height). 30 individuals were randomly selected by

136 picking random geographic coordinates and finding the closest individual at this location. For the

137 first tree sampled from each species, we clipped 50-100 g of leaves at the four cardinal points at

138 mid-canopy height, plus a single sample at bottom-canopy and top-canopy heights, into sterile

139 roll bags with surface-sterilized shears. We also sampled bottom-canopy leaves from two other

140 randomly chosen trees from each species. For bacterial community collection and amplification

141 we used the protocols described by Kembel et al. (2014). We collected microbial communities

142 from the leaf surface by five minutes of horizontal mechanical agitation of the samples in a

143 diluted Redford buffer solution. We resuspended cells in $500 \mu \mathrm{L}$ of PowerSoil bead solution

144 (MoBio, Carlsbad, California). We extracted DNA from isolated cells using the PowerSoil kit

145 according to the manufacturer's instructions and stored at $-80^{\circ} \mathrm{C}$. 


\section{DNA library preparation and sequencing}

148 We used a two-step PCR approach to prepare amplicon libraries for the high-throughput Illumina

149 sequencing platform. The use of combinatorial primers for paired-end Illumina sequencing of

150 amplicons reduced the number of primers while maintaining the diversity of unique identifiers

151 (Gloor et al. 2010). First, we amplified the V5-V6 region of the bacterial 16S rRNA gene using

152 chloroplast-excluding primers in order to eliminate contamination by host plant DNA (16S

153 primers 799F-1115R (Redford et al. 2010; Chelius \& Triplett 2001)) following protocols

154 described by Kembel et al. (2014). We cleaned the resulting product using MoBio UltraClean

155 PCR cleanup kit. We isolated a 445-bp fragment by electrophoresis in a $\%$ agarose gel, and

156 recovered DNA with the MoBio GelSpin kit. We prepared multiplexed 16S libraries by mixing

157 equimolar concentrations of DNA, and sequenced the DNA library using Illumina MiSeq 250-bp

158 paired-end sequencing at Genome Quebec.

159

160 We processed the raw sequence data with PEAR (Zhang et al. 2014) and QIIME

161 (Caporaso et al. 2010) software to merge paired-end sequences to a single sequence of length of

$162350 \mathrm{bp}$, eliminate low quality sequences (mean quality score $<30$ or with any series of 5 bases

163 with a quality score $<30$ ), and de-multiplex sequences into samples. We eliminated chimeric

164 sequences using the Uclust and Usearch algorithms (Edgar 2010). Then, we binned the

165 remaining sequences into operational taxonomic units (OTUs) at a 97\% sequence similarity

166 cutoff using the Uclust algorithm (Edgar 2010) and determined the taxonomic identity of each

167 OTU using the BLAST algorithm (Greengenes reference set) as implemented in QIIME

168 (Caporaso et al. 2010). The number of sequences per sample ranged from 6256 to 75 412. From 
169 these 1499777 sequences, we rarefied each sample to 5000 sequences and repeated analyses on

170100 random rarefactions. Re-analysis did not quantitatively change results and so we report only

171 the result of the analysis of a single random rarefaction. We included the resulting 275000

172 sequences in all subsequent analyses.

173

174 Statistical analyses

175 We created a database excluding OTUs represented fewer than 3 times to minimize the presence 176 of spurious OTUs caused by PCR and sequencing errors (Acinas et al. 2005). We identified the

177 OTUs that were present on all samples to define the "core microbiome" (Shade \& Handelsman,

178 2012). Then we tested for significant associations between bacterial taxa and host species, and

179 canopy location using the Linear Discriminant Analysis Effect Size (LEfSe) algorithm (Segata et

180 al. 2011). This analysis allows the recognition of significant individual host-microbe associations

181 and evaluates the strength of associations between organisms from different groups (Segata et al. 182 2011).

183

184 We performed analyses with the ape (Paradis et al. 2004), picante (Kembel et al. 2010), and 185 vegan (Oksanen et al. 2007) packages in R (R Development Core Team 2013) and ggplot2

186 (Wickham 2009) for data visualization. We quantified the taxonomic variation in bacterial

187 community structure among samples with respectively the Bray-Curtis dissimilarity. To illustrate

188 patterns of bacterial community structure, we performed a nonmetric multidimensional scaling

189 (NMDS) ordination of Bray-Curtis dissimilarity. We identified relationships between bacterial

190 community structure, host species identity, and sample canopy location by conducting a

191 permutational multivariate analysis of variance (PERMANOVA, Anderson 2001) on the 
192 community matrix. We employed a blocking randomization to account for the non-independence

193 of observations among sites. To decompose the total variation in the community matrix

194 explained by host species identity and canopy location, we performed a partial redundancy

195 analysis (RDA; Legendre \& Legendre 1998). This technique measures the amount of variation

196 that can be attributed exclusively to each set of explanatory variables. We performed three

197 permutational tests of multivariate homogeneity of group dispersions (Levene's test for

198 variances' homogeneity multivariate equivalent; Anderson 2006, Anderson et al. 2006): one to

199 test if variance in intra-individual canopy bacterial communities was equal between individuals

200 (30 samples from five trees sampled at six canopy locations); a second to compare interspecific

201 variation between species (30 bottom-canopy samples from 30 different trees); and finally a third

202 to test per-species intra- and inter-individual variation (all 55 samples). We estimated

203 phyllosphere bacterial alpha diversity using the Shannon index calculated from OTU relative

204 abundances for each community. We performed an analysis of variance (ANOVA) and

205 subsequent post-hoc Tukey's tests to compare differences in diversity across species. The

206 authors declare that the experiment comply with the current laws of the country in which the

207 experiment was performed.

208

209 RESULTS

210 Sequences, OTUs and taxonomy

211 High-throughput Illumina sequencing of the bacterial 16S rRNA gene (Claesson et al. 2010)

212 identified 5005 bacterial operational taxonomic units (OTUs, sequences binned at 97\%

213 similarity) in the phyllosphere of five temperate tree species, an average of $1055 \pm 57$ OTUs

214 (mean $\pm \mathrm{SE}$ ) per tree sampled. Most of these bacterial taxa were relatively common across 
215 samples, with only $3.4 \%$ of OTUs occurring on a single tree and $0.8 \%$ of OTUs occurring on all

216 trees. The OTUs present on all samples represent the "core microbiome": the microbial taxa

217 shared among multiple communities sampled from the same habitat (Shade \& Handelsman,

218 2012). In this study, the core microbiome consisted of 42 OTUs (Table 1) representing 61\% of

219 all sequences, of which 72\% were Alphaproteobacteria, 9\% Cytophagia, 7.8\%

220 Betaproteobacteria, 5\% Acidobacteria, 2\% Gammaproteobacteria and 2\% Actinobacteria. The

221 most abundant order was Rhizobiales (49\%) from which $77 \%$ of sequences were assigned to the

222 family Methylocystaceae. While there was some variation in the most abundant classes both

223 across the five tree species and among canopy locations (Figure 1 and 2), the class

224 Alphaproteobacteria was always the dominant taxon, with relative abundances ranging from

$22542 \%$ on $P$. glauca to $84 \%$ on B. papyrifera (Figure 1).

226

227 Intra-individual vs. Inter-Individual and Interspecific variation

228 Host species identity and individual identity effects could not be distinguished statistically due to

229 the fact that analyses of intra-individual variation were based on a single individual per species.

230 This host species/individual effect explained $65 \%$ of variation in phyllosphere bacterial

231 taxonomic community structure while the impact of canopy location was not statistically

232 significant (PERMANOVA on Bray-Curtis dissimilarities; Table 2). We then tested whether

233 canopy position had an effect on community structure after accounting for the variation

234 explained by host species/individual using a partial redundancy analysis (RDA) on bacterial

235 community structure constrained by host species identity. The RDA showed that when

236 differences in bacterial community structure driven by host species identity were accounted for,

237 sample canopy location explained $22 \%$ of the remaining variation in community structure. In 
238 comparison, in the dataset with 30 different individuals, host species identity explained only $47 \%$

239 of variation in phyllosphere bacterial community structure (PERMANOVA on Bray-Curtis

240 dissimilarities; Table 2). When considering intra-individual and inter-individual samples, host

241 species identity $\left(\mathrm{R}^{2}=47 \%\right)$ was the strongest driver of variation in phyllosphere bacterial

242 community structure closely followed by individual identity $\left(\mathrm{R}^{2}=32 \%\right)$ and finally by canopy

243 location $\left(\mathrm{R}^{2}=6 \%\right.$; PERMANOVA on Bray-Curtis dissimilarities; Table 2$)$. Community

244 composition of samples clustered based both on the individual (Figure 3a) and species (Figure

$2453 \mathrm{~b}$ ) from which they were collected (non-metric multidimensional scaling (NMDS) based on

246 Bray-Curtis distances among samples).

The first permutational multivariate test of variance homogeneity (an analogue of

Levene's test of homogeneity of variances) on intra-individual phyllosphere communities indicated a significant difference between P.glauca and B. papyrifera (Tukey's post hoc test; $\mathrm{P}=0.03$ ). The second test of the homogeneity of inter-individual variance between host species showed that $P$. glauca's variance in community structure (mean distance to centroid $=0.34$ ) was higher than A. saccharum $(0.25 ; \mathrm{P}<0.01)$ and $A$. rubrum $(0.26 ; \mathrm{P}<0.05)$ while all other comparisons were not significant. Finally, the third test between per species intra-individual and inter-individual variation indicated one significant difference in variation for B. papyrifera 256 ( $\mathrm{P}=0.005$; Figure 4). 
2610.04 for A. balsamea and $5.3 \pm$ SE 0.04 for $P$. glauca) than on angiosperm species $(3.7 \pm$ SE 0.06

262 for A. rubrum, $4.1 \pm$ SE 0.05 for A. saccharum and 3.6 \pm SE 0.09 for B. papyrifera).

263

264 Bacterial Indicator Taxa

265 The LEfSe analysis successfully identified indicator taxonomic groups associated with different

266 host species, but not across different canopy locations (Table 3). The conifers, A. balsamea and

267 P. glauca, had the highest number of associated bacterial indicator taxa (46 and 188

268 respectively). The strongest bio-indicators of A. balsamea were the Frankiaceae family and

269 multiple taxonomic levels of the phylum Acidobacteria: Acidobacteria, Acidobacteriales and

270 Acidobacteriaceae. For P. glauca, the strongest bioindicators were multiple taxa from the

271 Bacteroidetes phylum (Cytophagia, Cytophagales, Cytophagaceae, Spirosoma and Saprospirae,

272 Saprospirales, Chitinophagaceae), and from the Actinobacteria, Chloroflexi, and

273 Deltaproteobacteria. In contrast, B. papyrifera showed an overrepresentation of 24 bacterial taxa

274 including the phylum Proteobacteria, the class Alphaproteobacteria and several of its orders

275 (Rhodospiralles, Rickettsiales, Caulobacterales). Finally, the two Acer species (A. rubrum and

276 A. saccharum) were associated with 19 and 32 indicators respectively, including the order

277 Rhizobiales: A. rubrum being associated with the family Methylocystaceae and A. saccharum

278 with the order Methylobacteriaceae.

279

280 DISCUSSION

281 In this study, we demonstrate for multiple host species that there is a significant amount of intra-

282 individual variation in phyllosphere bacterial community structure (Figure 3a). While the mean

283 distance to centroid is always smaller for intra- than for inter-individual variation (Figure 4), this 
284 distance was only statistically significant for B. papyrifera. This result therefore provides partial

285 support for our first hypothesis, stating that magnitude of intra-individual variation would be

286 smaller than inter-individual and interspecific variation. When analyzing all samples, we found

287 host species identity to be a stronger determinant of phyllosphere bacterial community structure

288 than individual identity (Table 2). However, this result could be biased by the fact that we

289 sampled a single individual for multiple canopy location. The importance of host species identity

290 as a driver of phyllosphere community structure agrees with past studies of tropical (Kim et al.

291 2012; Kembel et al. 2014; Lambais et al. 2014) and temperate trees (Redford et al. 2010).

292 Previous studies have quantified intra- and inter-individual variation in phyllosphere bacterial

293 community structure, but these studies mixed leaves from within tree canopies without

294 quantifying intra-individual variation (Redford et al. 2010) or explored intra-individual variation

295 for a single host species (Leff et al. 2015). Our results show that after taking host species identity

296 into account, there exist detectable differences in microbial community structure within tree

297 canopies, at least in natural forest settings.

298

299

In terms of the taxonomic composition of the tree phyllosphere, each tree species can be

characterized by a particular combination of most abundant classes across all canopy locations, consistent with other studies of the phyllosphere microbiome (Redford et al. 2010; Kembel et al. species selective power on their phyllosphere bacterial communities, ecological strategies could

304 play a role by impacting leaf abiotic conditions. B. papyrifera, a shade intolerant species (Krajina et al. 1982; Burns \& Honkala 1990) exposed to sunlight in the upper part of the forest canopy,

306 exhibited the smallest alpha diversity with a dominance of Alphaproteobacteria (Figure 2e) and 
307 also the smallest amount of intra-individual variation (Figure 4). In contrast, both conifer host

308 species, growing below a deciduous canopy, exhibited the highest diversity in their community

309 structure. While ultraviolet radiation could be driving the observed differences in leaf alpha

310 diversity across species, our results provide no evidence of a significant and consistent difference

311 in the alpha-diversity among canopy locations. However, because we sampled only one

312 individual per species, canopy location effects remain to be quantified across multiple

313 individuals of the same species. As shown by the multivariate test of homogeneity of variance,

314 the intra-individual variation in phyllosphere community structure is not different from the

315 variation observed at the inter-individual level. Future phyllosphere studies characterizing the

316 relative influence of potential key factor such as random colonization via vectors such as the

317 atmospheric air flow (Barberán et al. 2014) or animals (Scheffers et al. 2013), competition

318 between bacterial populations (Vorholt 2012); or intra-individual variation in leaf functional

319 traits (Hunter et al. 2010; Reisberg et al. 2012) are needed to understand the dynamics driving

320 intra-individual variability in bacterial community structure.

In conclusion, our results demonstrate that there exists considerable intra-individual

323 variation in phyllosphere community structure, and that the magnitude of this variation is smaller

324 but not statistically different from the magnitude of inter-individual variation. When designing a

325 study of tree phyllosphere bacterial communities, if quantifying interspecific variation is the goal

326 then samples from a consistent location within the tree canopy for individual trees are sufficient

327 to quantify the majority of the variation in community structure. However, future studies and

328 especially studies focusing on a single host species should acknowledge that there can be

329 significant intra-individual variation in phyllosphere community structure, and sampling plans 
330 should explicitly select leaves at different positions within the canopy to describe spatial

331 structure of the overall community composition for individual trees. 


\section{AVAILABILITY OF THE DATA AND MATERIALS}

333 The datasets supporting the conclusions of this article are available on Figshare at:

334 https://figshare.com/s/45794d675d912b83a2f2 (code in R),

335 https://figshare.com/s/bd133cfbd2ca0cf8f449 (OTU table),

336 https://figshare.com/s/be5c5ed6b224667e8fd1 (barcodes),

337 https://figshare.com/s/d1703c191b2c0f4f54db (metadata) and

338 https://figshare.com/s/b93099c2ea930c5d6553 (16S rRNA sequences).

339

340 ACKNOWLEDGEMENTS

341 We thank Travis Dawson, Sophie Carpentier and Gabriel Jacques for support in the field and 342 laboratory. 


\section{REFERENCES}

344 Acinas, S. G., Sarma-Rupavtarm, R., Klepac-Ceraj, V., \& Polz, M. F. (2005). PCR-induced sequence artifacts and bias: insights from comparison of two 16S rRNA clone libraries constructed from the same sample. Applied and environmental microbiology, 71(12), 8966-8969.

Allison, S. D., \& Treseder, K. K. (2011). Climate change feedbacks to microbial decomposition in boreal soils. Fungal Ecology, 4(6):362-374.

Anderson, M.J. (2001). A new method for non-parametric multivariate analysis of variance. Austral ecology, 26(1):32-46.

352

353

Anderson, M.J. (2006) Distance-based tests for homogeneity of multivariate dispersions. Biometrics 62(1):245-253.

Anderson, M.J., Ellingsen, K.E. \& McArdle, B.H. (2006) Multivariate dispersion as a measure of beta diversity. Ecology Letters 9(6):683-693.

Atamna-Ismaeel N, Finkel O, Glaser F, von Mering C, Vorholt JA, Koblížek M, Belkin S, \& Béjà O. (2012) Bacterial anoxygenic photosynthesis on plant leaf surfaces. Environmental microbiology reports. 4(2):209-16.

Barberán, A., Ladau, J., Leff, J. W., Pollard, K. S., Menninger, H. L., Dunn, R. R., \& Fierer, N. (2015). Continental-scale distributions of dust-associated bacteria and fungi. Proceedings of the National Academy of Sciences, 112(18), 5756-5761.

Bodenhausen, N., Bortfeld-Miller, M., Ackermann, M., \& Vorholt, J. A. (2014). A synthetic community approach reveals plant genotypes affecting the phyllosphere microbiota. PLoS Genet, 10(4):e1004283. 
365 Bradley, D. J., Gilbert, G. S., \& Martiny, J. B. (2008). Pathogens promote plant diversity through

366 a compensatory response. Ecology Letters, 11(5):461-469.

367 Burns, R. M., \& Honkala, B. H. (1990). Silvics of North America. Vol. 1. Conifers. US Dep.

$368 \quad$ Agric. Agric. Handb, 654.

369 Caporaso, J.G., Kuczynski, J., Stombaugh, J., Bittinger, K., Bushman, F.D., Costello, E.K.,

370 Fierer, N., Pena, A.G., Goodrich, J.K., Gordon, J.I. \& Huttley, G.A. (2010). QIIME

371 allows analysis of high-throughput community sequencing data. Nature methods, 7(5),

$372 \quad 335-336$.

373 Chelius, M. K., \& Triplett, E. W. (2001). The Diversity of Archaea and Bacteria in Association with the Roots of Zea mays L. Microbial Ecology, 41(3), 252-263.

375

15. Claesson, M.J., Wang, Q., O'Sullivan, O., Greene-Diniz, R., Cole, J.R., Ross, R.P. \& 376 O'Toole, P.W. (2010) Comparison of two next-generation sequencing technologies for resolving highly complex microbiota composition using tandem variable $16 \mathrm{~S}$ rRNA gene regions. Nucleic Acids Research 38(22):e200-e200.

379 Clay, K., \& Holah, J. (1999). Fungal endophyte symbiosis and plant diversity in successional fields. Science, 285(5434) :1742-1744.

381 Cordier, T., Robin, C., Capdevielle, X., Desprez-Loustau, M. L., \& Vacher, C. (2012a). Spatial variability of phyllosphere fungal assemblages: genetic distance predominates over geographic distance in a European beech stand (Fagus sylvatica). Fungal ecology, 5(5):509-520.

385 Cordier, T., Robin, C., Capdevielle, X., Fabreguettes, O., Desprez-Loustau, M. L., \& Vacher, C. (2012b). The composition of phyllosphere fungal assemblages of European beech (Fagus 
sylvatica) varies significantly along an elevation gradient. New Phytologist, 196(2), 510519.

Edgar, R. C. (2010). Search and clustering orders of magnitude faster than BLAST. Bioinformatics, 26(19), 2460-2461.

391 Finkel, O. M., Burch, A. Y., Lindow, S. E., Post, A. F., \& Belkin, S. (2011). Geographical location determines the population structure in phyllosphere microbial communities of a salt-excreting desert tree. Applied and environmental microbiology, 77(21):7647-7655.

394 Finkel, O. M., Burch, A. Y., Elad, T., Huse, S. M., Lindow, S. E., Post, A. F., \& Belkin, S.

395

396

397

398

400

401

402

403 (2012). Distance-decay relationships partially determine diversity patterns of phyllosphere bacteria on Tamrix trees across the Sonoran Desert. Applied and environmental microbiology, 78(17):6187-6193.

Gloor, G. B., Hummelen, R., Macklaim, J. M., Dickson, R. J., Fernandes, A. D., MacPhee, R., \& Reid, G. (2010). Microbiome profiling by illumina sequencing of combinatorial sequence-tagged PCR products. PloS one, 5(10), e15406.

Grace, J., Malcolm, D. C., \& Bradbury, I. K. (1975). The effect of wind and humidity on leaf diffusive resistance in Sitka spruce seedlings. Journal of Applied Ecology, 931-940.

Horton, M.W., Bodenhausen, N., Beilsmith, K., Meng, D., Muegge, B.D., Subramanian, S., Vetter, M.M., Vilhjálmsson, B.J., Nordborg, M., Gordon, J.I. \& Bergelson, J., 2014. Genome-wide association study of Arabidopsis thaliana leaf microbial community. Nature communications, 5 .

Hunter, P. J., Hand, P., Pink, D., Whipps, J. M., \& Bending, G. D. (2010). Both leaf properties and microbe-microbe interactions influence within-species variation in bacterial 
population diversity and structure in the lettuce (Lactuca species) phyllosphere. Applied and environmental microbiology, 76(24), 8117-8125.

411 Inácio, J., Pereira, P., Carvalho, D. M., Fonseca, A., Amaral-Collaco, M. T., \& Spencer-Martins, I. (2002). Estimation and diversity of phylloplane mycobiota on selected plants in a mediterranean-type ecosystem in Portugal. Microbial Ecology, 44(4), 344-353.

414 Innerebner, G., Knief, C., \& Vorholt, J. A. (2011). Protection of Arabidopsis thaliana against leaf-pathogenic Pseudomonas syringae by Sphingomonas strains in a controlled model system. Applied and environmental microbiology, 77(10), 3202-3210.

417

418

Jackson, C. R., \& Denney, W. C. (2011). Annual and seasonal variation in the phyllosphere bacterial community associated with leaves of the southern magnolia (Magnolia grandiflora). Microbial ecology, 61(1), 113-122.

Jumpponen, A., \& Jones, K. L. (2009). Massively parallel 454 sequencing indicates hyperdiverse fungal communities in temperate Quercus macrocarpa phyllosphere. New Phytologist, 184(2), 438-448.

Jumpponen, A., \& Jones, K. L. (2010). Seasonally dynamic fungal communities in the Quercus macrocarpa phyllosphere differ between urban and nonurban environments. $\mathrm{New}$ Phytologist, 186(2), 496-513.

Kadivar, H., \& Stapleton, A. E. (2003). Ultraviolet radiation alters maize phyllosphere bacterial diversity. Microbial ecology, 45(4), 353-361.

Kembel, S.W., Cowan, P.D., Helmus, M.R., Cornwell, W.K., Morlon, H., Ackerly, D.D., Blomberg, S.P. \& Webb, C.O. (2010). Picante: R tools for integrating phylogenies and ecology. Bioinformatics, 26(11), 1463-1464. 
431 Kembel, S. W., \& Mueller, R. C. (2014). Plant traits and taxonomy drive host associations in 432 tropical phyllosphere fungal communities 1. Botany, 92(4):303-311.

433 Kembel, S. W., O’Connor, T. K., Arnold, H. K., Hubbell, S. P., Wright, S. J., \& Green, J. L.

434 (2014). Relationships between phyllosphere bacterial communities and plant functional

435 traits in a neotropical forest. Proceedings of the National Academy of Sciences,

$436 \quad 111(38): 13715-13720$.

437 Kim, M., Singh, D., Lai-Hoe, A., Go, R., Rahim, R.A., Ainuddin, A.N., Chun, J., \& Adams, J.M.

438

439 (2012). Distinctive phyllosphere bacterial communities in tropical trees. Microbial ecology, 63(3), 674-681.

Krajina, V. J., Klinka, K., \& Worrall, J. (1982). Distribution and ecological characteristics of trees and shrubs of British Columbia. University of British Columbia, Faculty of Forestry.

443 444
Laforest-Lapointe, I., Messier, C. \& Kembel, S. W. (2016) Host species identity, site and time drive temperate tree phyllosphere bacterial community structure. Microbiome, 4(1)-1.

Lambais, M. R., Crowley, D. E., Cury, J. C., Büll, R. C., \& Rodrigues, R. R. (2006). Bacterial diversity in tree canopies of the Atlantic forest. Science, 312(5782), 1917-1917.

Lambais, M. R., Lucheta, A. R., \& Crowley, D. E. (2014). Bacterial community assemblages associated with the phyllosphere, dermosphere, and rhizosphere of tree species of the Atlantic forest are host taxon dependent. Microbial ecology, 68(3), 567-574.

Leff, J. W., Del Tredici, P., Friedman, W. E., \& Fierer, N. (2015). Spatial structuring of bacterial communities within individual Ginkgo biloba trees. Environmental microbiology, 17(7), 2352-2361. 
453 Legendre, P., \& Legendre, L. (1998). Numerical ecology: second English edition. Developments $454 \quad$ in environmental modelling, 20.

455 Leveau, J. H., \& Lindow, S. E. (2001). Appetite of an epiphyte: quantitative monitoring of 456 bacterial sugar consumption in the phyllosphere. Proceedings of the National Academy of

Lichtenthaler, H.K., Buschmann, C., Döll, M., Fietz, H.J., Bach, T., Kozel, U., Meier, D., \& Sciences, 98(6), 3446-3453. Rahmsdorf, U. (1981). Photosynthetic activity, chloroplast ultrastructure, and leaf characteristics of high-light and low-light plants and of sun and shade leaves. Photosynthesis research, 2(2), 115-141.

Lindow, S. E., \& Brandl, M. T. (2003). Microbiology of the phyllosphere. Applied and environmental microbiology, 69(4), 1875-1883.

464 McGuire, K. L., \& Treseder, K. K. (2010). Microbial communities and their relevance for ecosystem models: decomposition as a case study. Soil Biology and Biochemistry, 42(4), $529-535$.

Medina-Martínez, M. S., Allende, A., Barberá, G. G., \& Gil, M. I. (2015). Climatic variations 468 influence the dynamic of epiphyte bacteria of baby lettuce. Food Research International, $68,54-61$.

Miller, W. G., Brandl, M. T., Quiñones, B., \& Lindow, S. E. (2001). Biological sensor for sucrose availability: relative sensitivities of various reporter genes. Applied and environmental microbiology, 67(3):1308-1317.

473 Oksanen, J., Kindt, R., Legendre, P., O’Hara, B., Stevens, M. H. H., Oksanen, M. J., \& Suggests, 474 M. A. S. S. (2007) The vegan package. Community ecology package. 
475 Paradis E, Claude J, \& Strimmer K. (2004) APE: analyses of phylogenetics and evolution in R 476 language. Bioinformatics, 20:289-290.

477 Penuelas, J., Rico, L., Ogaya, R., Jump, A. S., \& Terradas, J. (2012). Summer season and

478 long-term drought increase the richness of bacteria and fungi in the foliar phyllosphere of 479 Quercus ilex in a mixed Mediterranean forest. Plant Biology, 14(4), 565-575.

480 R Development Core Team. R: A language and Environment for Statistical Computing. Vienna, 481 Austria. 2013. URL http://www.R-project.org/.

482 Redford, A. J., \& Fierer, N. (2009). Bacterial succession on the leaf surface: a novel system for 483 studying successional dynamics. Microbial ecology, 58(1), 189-198.

484 Redford, A. J., Bowers, R. M., Knight, R., Linhart, Y., \& Fierer, N. (2010). The ecology of the 485 phyllosphere: geographic and phylogenetic variability in the distribution of bacteria on tree leaves. Environmental microbiology, 12(11), 2885-2893.

487

488

489

490

491

492

493

494

495

496

Reisberg, E. E., Hildebrandt, U., Riederer, M., \& Hentschel, U. (2012). Phyllosphere bacterial communities of trichome-bearing and trichomeless Arabidopsis thaliana leaves. Antonie Van Leeuwenhoek, 101(3), 551-560.

Ritpitakphong U, Falquet L, Vimoltust A, Berger A, Métraux JP, \& L'Haridon F. (2016) The microbiome of the leaf surface of Arabidopsis protects against a fungal pathogen. New Phytologist.

Rodriguez, R. J., White Jr, J. F., Arnold, A. E., \& Redman, R. S. (2009). Fungal endophytes: diversity and functional roles. New phytologist, 182(2), 314-330.

Scheffers, B. R., Phillips, B. L., Laurance, W. F., Sodhi, N. S., Diesmos, A., \& Williams, S. E. (2013). Increasing arboreality with altitude: a novel biogeographic dimension. 

20131581.

499 Segata, N., Izard, J., Waldron, L., Gevers, D., Miropolsky, L., Garrett, W. S., \& Huttenhower, C.

500 (2011). Metagenomic biomarker discovery and explanation.Genome Biol, 12(6), R60.

501 Shade, A., \& Handelsman, J. (2012). Beyond the Venn diagram: the hunt for a core microbiome. $502 \quad$ Environmental microbiology, 14(1), 4-12.

503 Van Der Heijden, M. G., Bardgett, R. D., \& Van Straalen, N. M. (2008). The unseen majority: 504 soil microbes as drivers of plant diversity and productivity in terrestrial ecosystems.

$505 \quad$ Ecology letters, 11(3), 296-310.

506 Vorholt, J. A. (2012). Microbial life in the phyllosphere. Nature Reviews Microbiology, 10(12), $507 \quad 828-840$.

508 Wickham H. ggplot2: Elegant Graphics for Data Analysis. Springer New York; 2009.

509 Zhang, J., Kobert, K., Flouri, T., \& Stamatakis, A. (2014). PEAR: a fast and accurate Illumina 510 Paired-End reAd mergeR. Bioinformatics, 30(5), 614-620. 


\section{FIGURE LEGENDS}

513

514 FIGURE 1. Relative abundance of sequences from bacterial taxonomic classes in the

515 phyllosphere microbiome of temperate tree species in a Quebec forest. (ABBA: Abies balsamea;

516 ACRU: Acer rubrum; ACSA: Acer saccharum; BEPA: Betula papyrifera; PIGL: Picea glauca).

517 FIGURE 2. Relative abundance of bacterial classes in the phyllosphere at six canopy locations

518 (B:Bottom, E:East, N:North, W:West, S:South T:Top) for one individual of the five temperate

519 tree species under study. a) Abies balsamea; b) Picea glauca; c) Acer rubrum; d) Acer

520 saccharum; and e) Betula papyrifera.

521 FIGURE 3. Non-metric multidimensional scaling (NMDS) ordination of within-individual

522 variation in bacterial community structure across 55 phyllosphere samples from Quebec

523 temperate forest trees. Ellipses indicate 1 standard deviation confidence interval around of a)

524 intra-individual samples and b) inter-individual samples. Gray boxes indicate the 30 samples that 525 came from individuals sampled at six different canopy locations. The other 25 samples came 526 from 5 more individuals per host species. Symbols indicate sample position in the tree canopy;

527 colours indicate by host species identity (green: Abies balsamea; red: Acer rubrum; orange: Acer 528 saccharum; purple: Betula papyrifera; blue: Picea glauca). Stress value was 0.16.

529 FIGURE 4. Permutation test for homogeneity of multivariate dispersions in leaf bacterial 530 communities between per species intra- and inter-individual samples. Colours indicate host

531 species identity (green for Abies balsamea; red for Acer rubrum; orange for Acer saccharum;

532 purple for Betula papyrifera; and blue for Picea glauca); shading indicate intra- (pale color) and 533 inter-individual (dark color) variance respectively. 


\section{TABLE LEGENDS}

535

536 TABLE 1. Taxonomy and relative abundance of the 42 OTUs constituting the tree phyllosphere

537 bacterial core microbiome in Quebec temperate forests (present in all 55 samples).

538

539 TABLE 2. Variation in phyllosphere bacterial community structure explained by various drivers:

540 host species identity, sample location within the tree canopy and individual identity.

541 PERMANOVA on Bray-Curtis dissimilarities.

542

543 TABLE 3. Bacterial taxa identified as bio-indicators of different host species in Quebec

544 temperate forests. The LEfSe analysis was performed on 30 samples: 6 individuals per species.

545 Only the top five bio-indicators are shown. Significance are given by: $* \mathrm{P}<0.05 ; * * \mathrm{P}<0.01$;

$546 * * * \mathrm{P}<0.001 ; \mathrm{NS}, \mathrm{P}>0.05$. 


\section{Figure 1}

Relative abundance of sequences from bacterial taxonomic classes in the phyllosphere microbiome of temperate tree species in a Quebec forest.

(ABBA: Abies balsamea; ACRU: Acer rubrum; ACSA: Acer saccharum; BEPA: Betula papyrifera; PIGL: Picea glauca).

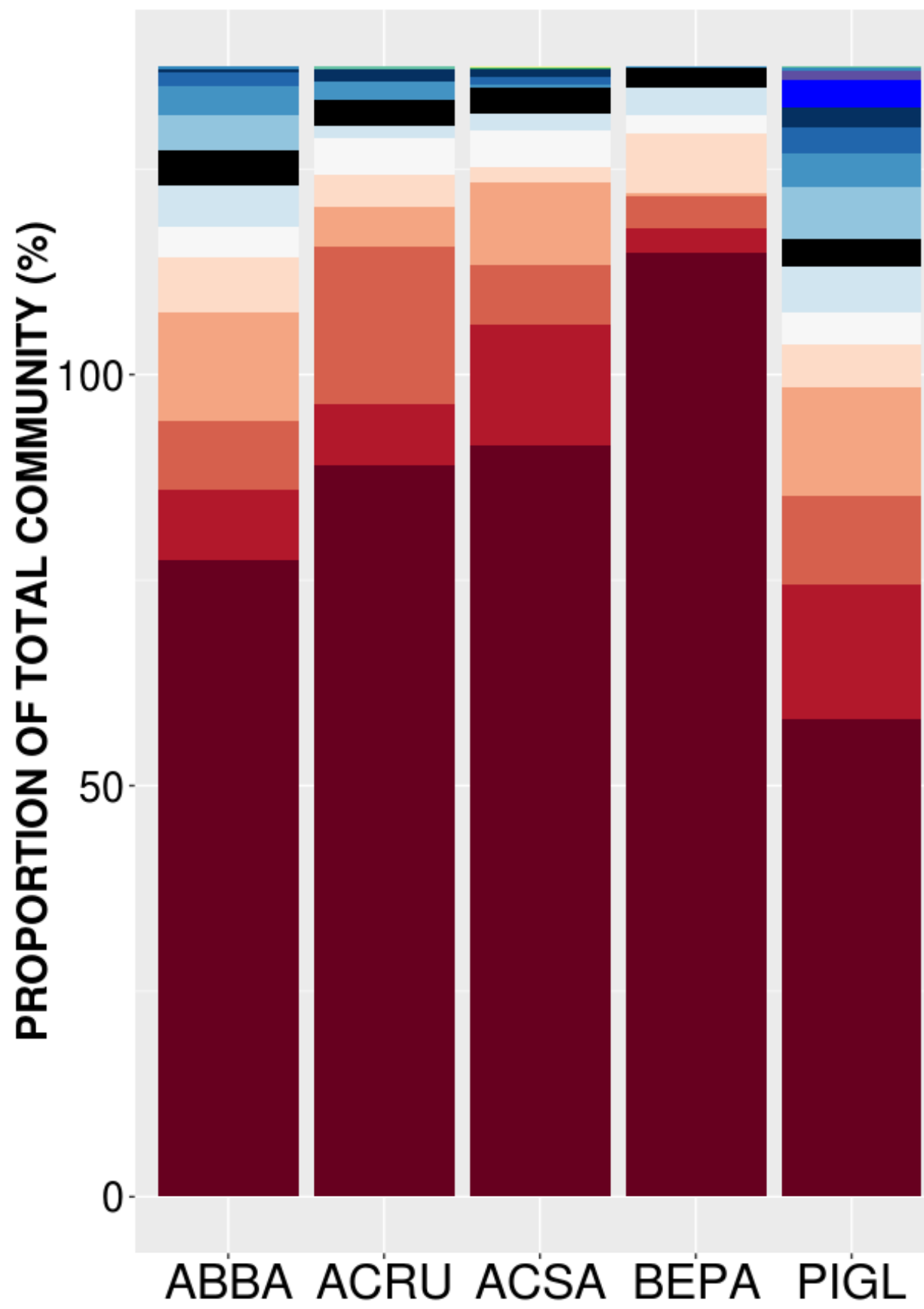

BACTERIAL CLASS

Alphaproteobacteria Cytophagia Betaproteobacteria Actinobacteria Acidobacteriia Gammaproteobacteria Deltaproteobacteria

Other [Saprospirae] Sphingobacteriia Deinococci

TM7-3

Chloroflexi Thermoleophilia Armatimonadia Flavobacteriia ABS-6 DA052 
Figure 2

Relative abundance of bacterial classes in the phyllosphere at 6 canopy locations (B:Bottom, E:East, N:North, W:West, S:South T:Top) for one individual of the five temperate tree species under study.

a) Abies balsamea; b) Picea glauca; c) Acer rubrum; d) Acer saccharum; and e) Betula papyrifera. 

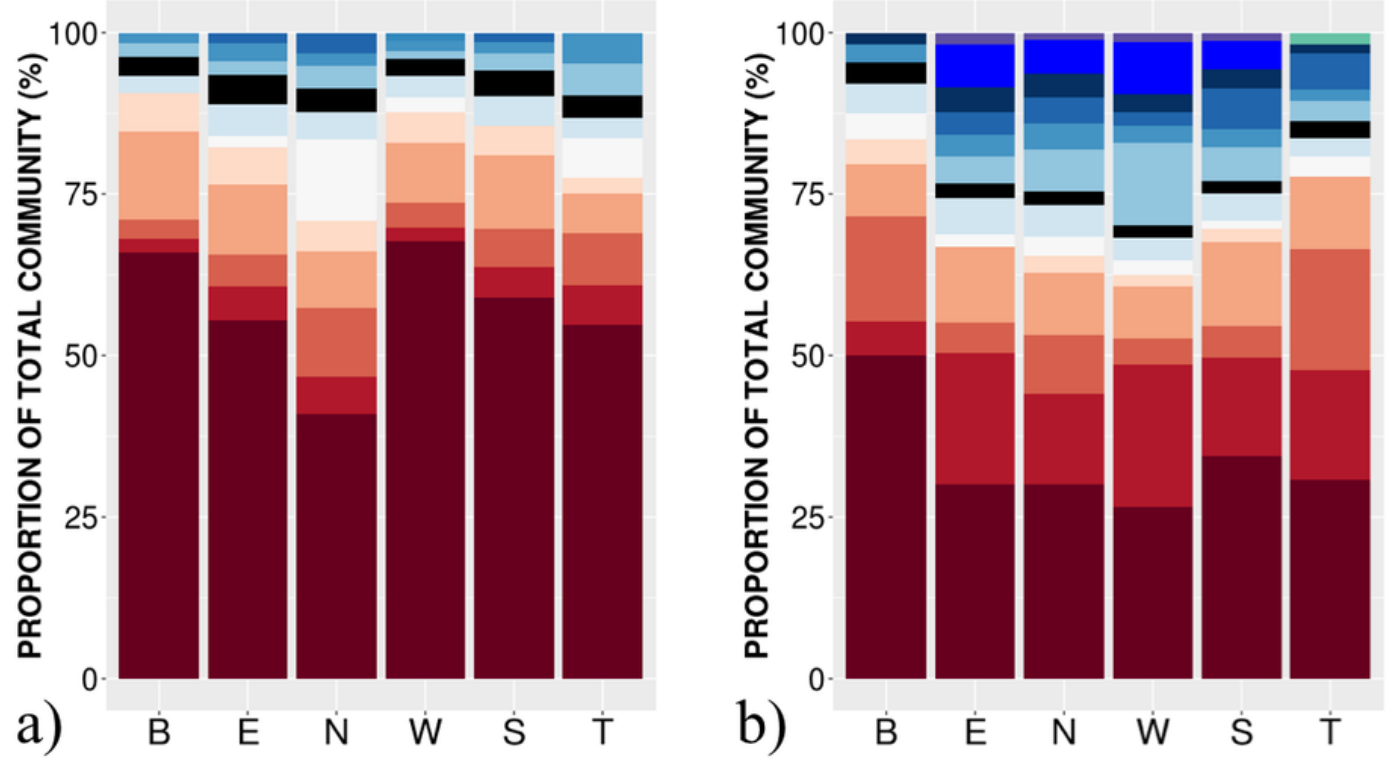

b) $\begin{array}{llllll}B & E & N & W & S & \top\end{array}$
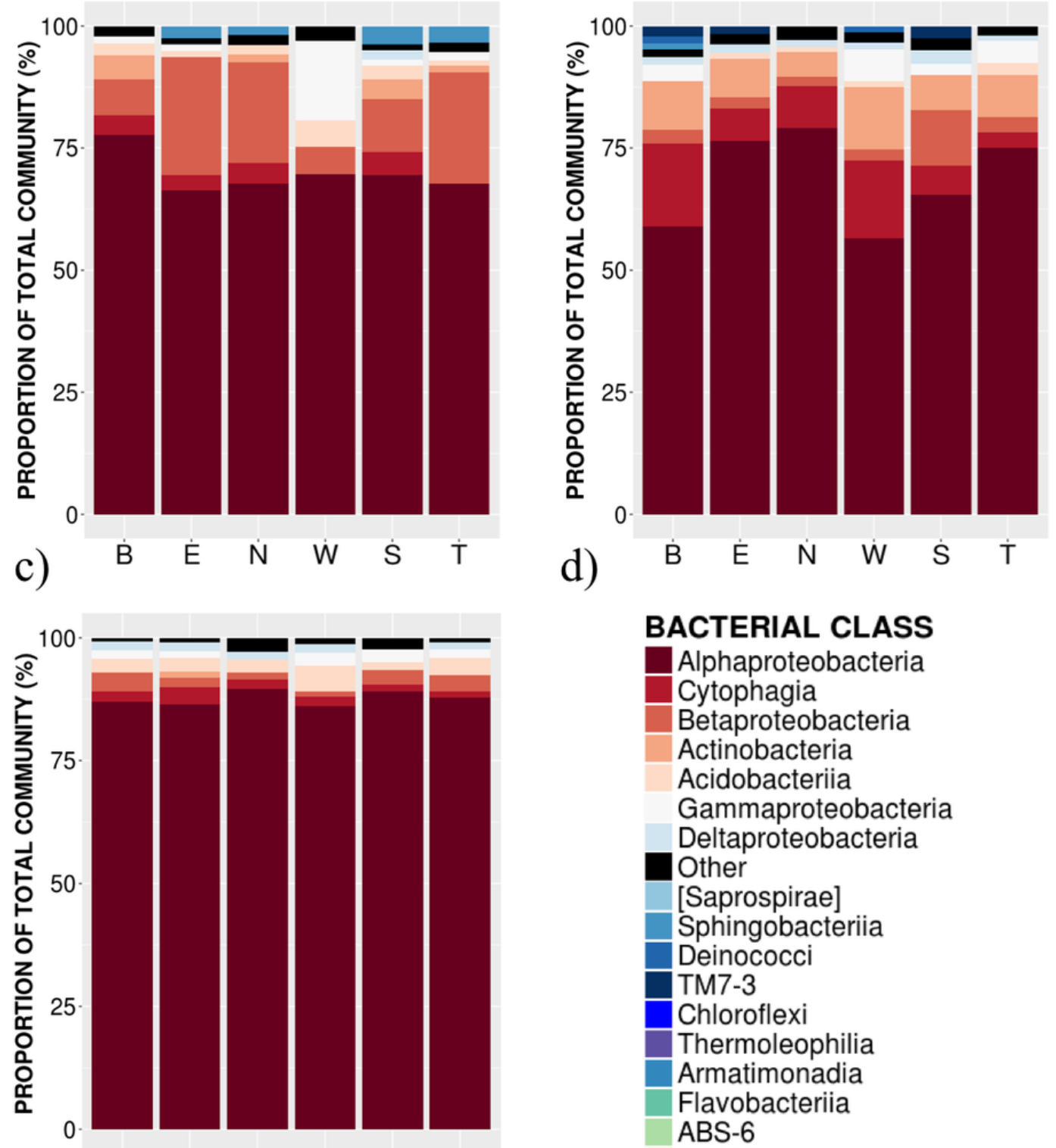

BACTERIAL CLASS

Alphaproteobacteria

Cytophagia

Betaproteobacteria

Actinobacteria

Acidobacteriia

Gammaproteobacteria

Deltaproteobacteria

Other

[Saprospirae]

Sphingobacteriia

Deinococci

TM7-3

Chloroflexi

Thermoleophilia

Armatimonadia

Flavobacteriia

ABS-6

DA052

e) $\frac{\text { B }}{\text { Peer) reviewing PDF | (2016:05:10568:1:0:NEW } 11 \text { Jul 2016) }}$ 


\section{Figure 3}

Non-metric multidimensional scaling (NMDS) ordination of within-individual variation in bacterial community structure across 55 phyllosphere samples from Quebec temperate forest trees.

Stress amounted to 0.16 . Ellipses indicate 1 standard deviation confidence interval around of

a) intra-individual samples and b) inter-individual samples. Gray boxes indicate the 30 samples that came from individuals sampled at six different canopy locations. The other 25 samples came from 5 more individuals per host species. Symbols indicate sample position in the tree canopy; colours indicate by host species identity (green: Abies balsamea; red: Acer rubrum; orange: Acer saccharum; purple: Betula papyrifera; blue: Picea glauca). 

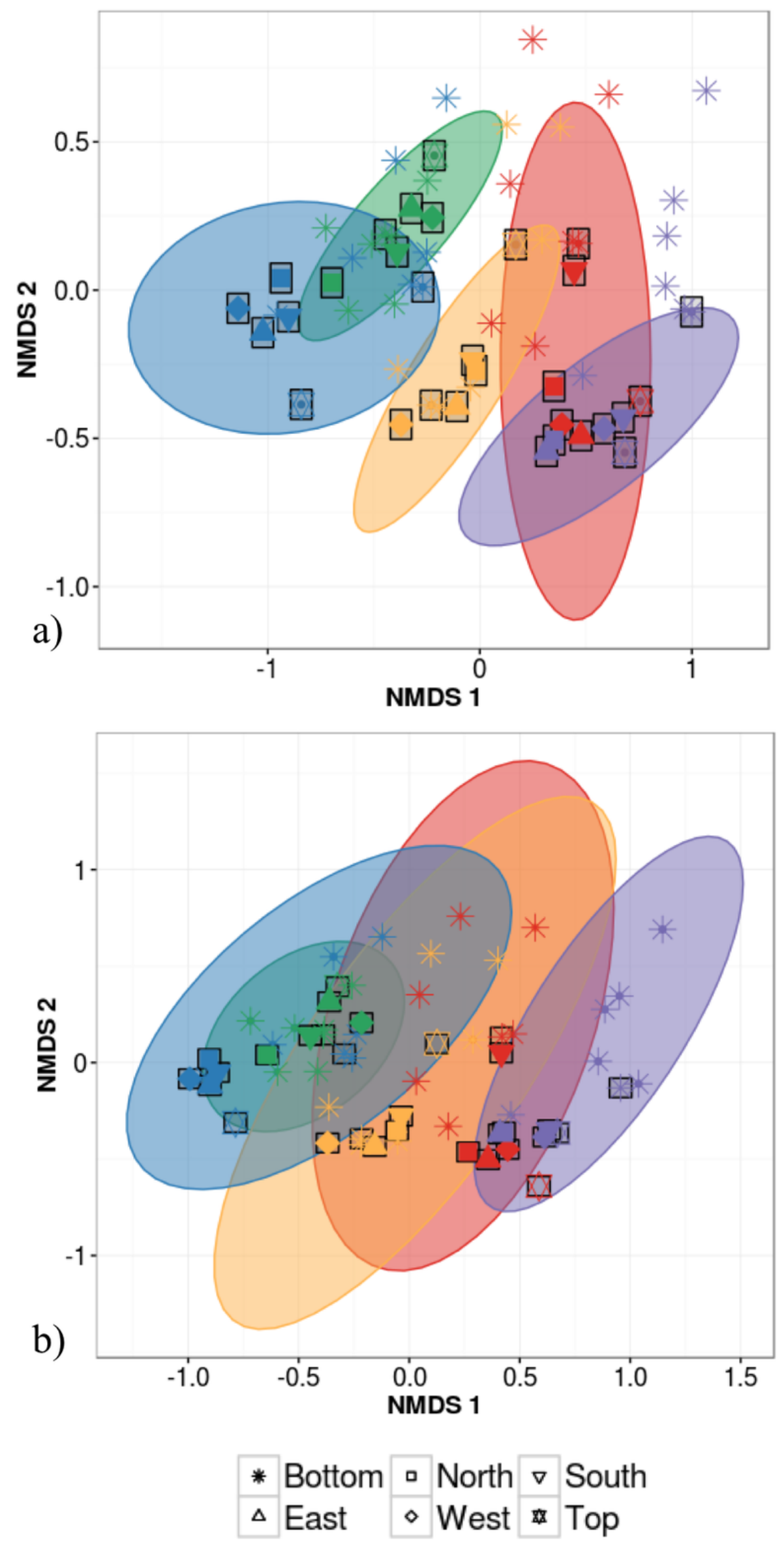


\section{Figure 4}

Permutation test for homogeneity of multivariate dispersions in leaf bacterial communities between per species intra- and inter-individual samples.

Colours indicate host species identity (green for Abies balsamea; red for Acer rubrum; orange for Acer saccharum; purple for Betula papyrifera; and blue for Picea glauca); shading indicate intra- (pale color) and inter-individual (dark color) variance respectively.

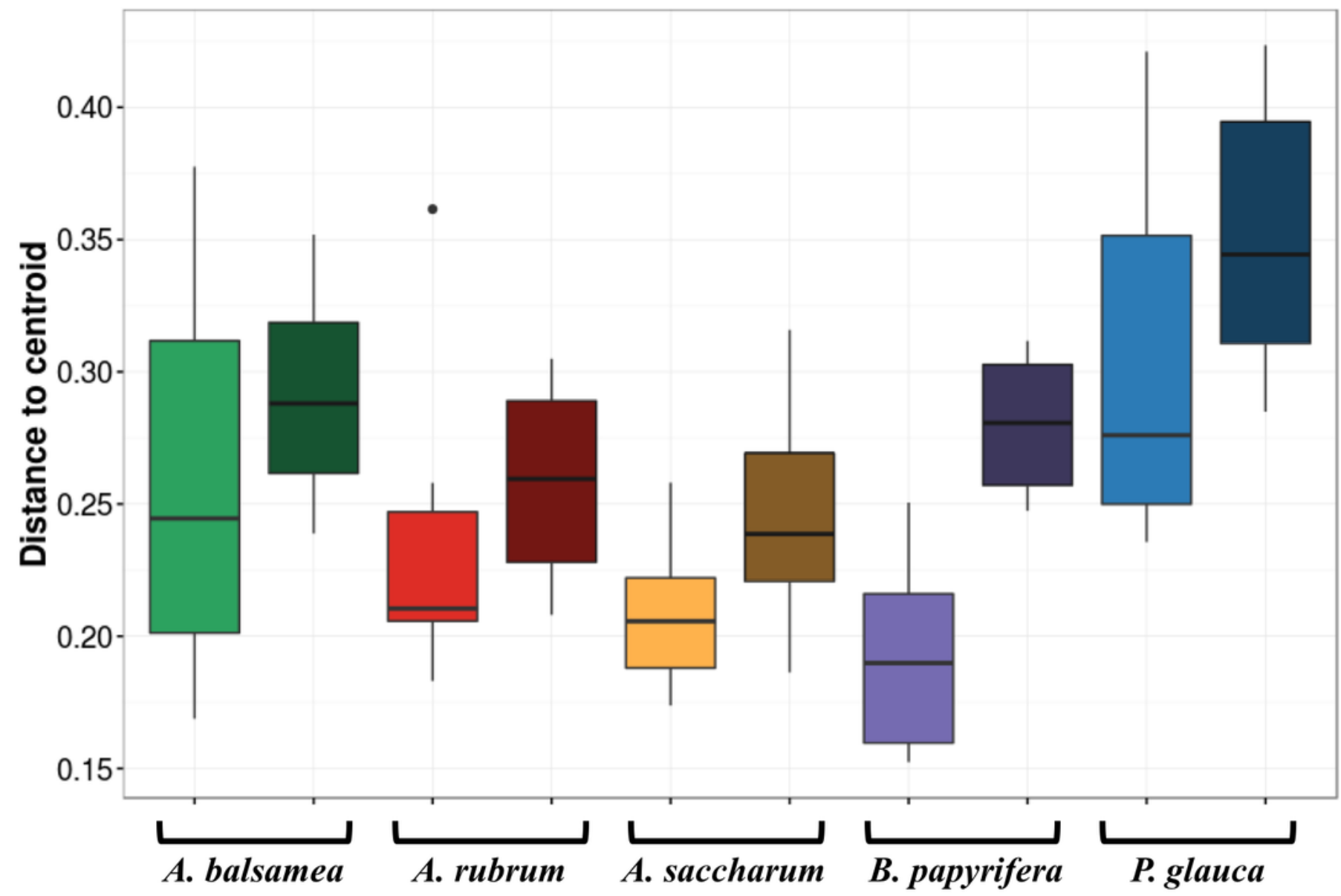




\section{Table $\mathbf{1}$ (on next page)}

Taxonomy and relative abundance of the 42 OTUs constituting the tree phyllosphere bacterial core microbiome in Quebec temperate forests (present in all 55 samples). 


\begin{tabular}{|c|c|c|c|c|c|}
\hline CLASS & ORDER & FAMILY & GENERA & SPECIES & $\%$ \\
\hline \multirow{2}{*}{ Acidobacteriia } & \multirow{2}{*}{ Acidobacteriales } & \multirow{2}{*}{ Acidobacteriaceae } & Bryocella & elongata & 0.5 \\
\hline & & & \multicolumn{2}{|c|}{$4 \mathrm{NAs}$} & 4.8 \\
\hline \multirow{2}{*}{ Actinobacteria } & \multirow{2}{*}{ Actinomycetales } & Frankiaceae & \multicolumn{2}{|c|}{ NA } & 1.3 \\
\hline & & Microbacteriaceae & Frondihabitans & cladoniiphilus & 0.5 \\
\hline Cytophagia & Cytophagales & Cytophagaceae & Hymenobacter & 2 NAs & 9.0 \\
\hline \multirow{2}{*}{ Sphingobacteriia } & \multirow{2}{*}{ Sphingobacteriales } & \multirow{2}{*}{ Sphingobacteriaceae } & Mucilaginibacter & daejeonensis & 0.5 \\
\hline & & & \multicolumn{2}{|c|}{ NA } & 0.2 \\
\hline \multirow{10}{*}{ Alphaproteobacteria } & Caulobacterales & Caulobacteraceae & \multicolumn{2}{|c|}{ NA } & 1.5 \\
\hline & \multirow{3}{*}{ Rhizobiales } & Beijerinckiaceae & Beijerinckia & 2 NAs & 8.9 \\
\hline & & Methylobacteriaceae & Methylobacterium & 2 NAs & 2.3 \\
\hline & & Methylocystaceae & \multicolumn{2}{|c|}{7 NAs } & 38.1 \\
\hline & Rhodospirillales & Acetobacteraceae & \multicolumn{2}{|c|}{6 NAs } & 11.2 \\
\hline & \multirow{2}{*}{ Rickettsiales } & NA & \multicolumn{2}{|c|}{ NA } & 0.10 \\
\hline & & Rickettsiaceae & Rickettsia & NA & 0.6 \\
\hline & \multirow{3}{*}{ Sphingomonadales } & \multirow{3}{*}{ Sphingomonadaceae } & \multirow{3}{*}{ Sphingomonas } & 6 NAs & 7.9 \\
\hline & & & & wittichii & 1.7 \\
\hline & & & & wittichii & 0.1 \\
\hline Betaproteobacteria & Burkholderiales & Oxalobacteraceae & \multicolumn{2}{|c|}{2 NAs } & 7.8 \\
\hline \multirow{2}{*}{ Deltaproteobacteria } & Bdellovibrionales & Bdellovibrionaceae & Bdellovibrio & $\mathrm{NA}$ & 0.2 \\
\hline & Myxococcales & Cystobacterineae & \multicolumn{2}{|l|}{ NA } & 0.7 \\
\hline \multirow{2}{*}{ Gammaproteobacteria } & Enterobacteriales & Enterobacteriaceae & Erwinia & NA & 0.7 \\
\hline & Pseudomonadales & Pseudomonadaceae & Pseudomonas & fragi & 1.3 \\
\hline
\end{tabular}




\section{Table 2 (on next page)}

Variation in phyllosphere bacterial community structure explained by various drivers: host species identity, sample location within the tree canopy and individual identity.

PERMANOVA on Bray-Curtis dissimilarities. 


\begin{tabular}{|c|c|c|c|c|c|c|}
\hline \multirow[b]{2}{*}{ Dataset } & \multirow[b]{2}{*}{ Scope } & \multirow[b]{2}{*}{$\begin{array}{c}\mathrm{Nb} \\
\text { samples }\end{array}$} & \multirow[b]{2}{*}{$\begin{array}{c}\mathrm{Nb} \\
\text { ind./species }\end{array}$} & \multicolumn{3}{|c|}{ Variables $R^{2}(\%)$} \\
\hline & & & & $\begin{array}{l}\text { Canopy } \\
\text { location }\end{array}$ & $\begin{array}{c}\text { Host } \\
\text { species } \\
\text { identity } \\
\end{array}$ & $\begin{array}{c}\text { Individual } \\
\text { identity }\end{array}$ \\
\hline$\# 1$ & Intra-individual & 30 & 1 & $8^{*}$ & & \\
\hline$\# 2$ & $\begin{array}{l}\text { Inter-individual and } \\
\text { interspecific }\end{array}$ & 30 & 6 & $n a$ & 47 & $n a$ \\
\hline$\# 3$ & $\begin{array}{l}\text { Intra- and inter- } \\
\text { individual, and } \\
\text { interspecific }\end{array}$ & 60 & 6 & 6 & 47 & $32^{* * * *}$ \\
\hline
\end{tabular}




\section{Table 3(on next page)}

Bacterial taxa identified as bio-indicators of different host species in Quebec temperate forests.

The LEfSe analysis was performed on 30 samples: 6 individuals per species. Only the top five bio-indicators are shown. Significance are given by: $* \mathrm{P}<0.05$; $* * \mathrm{P}<0.01$; *** $\mathrm{P}<0.001$; NS, $\mathrm{P}>0.05$. 


\begin{tabular}{|c|c|c|}
\hline $\begin{array}{c}\text { HOST } \\
\text { SPECIES } \\
\text { IDENTITY }\end{array}$ & BACTERIAL TAXA & $\begin{array}{c}\text { EFFECT } \\
\text { SIZE }\end{array}$ \\
\hline \multirow{5}{*}{$\begin{array}{c}\text { Abies } \\
\text { balsamea }\end{array}$} & Actinobacteria.Actinobacteria.Actinomycetales.Frankiaceae & $4.34 * * *$ \\
\hline & Acidobacteria & $4.30 * * *$ \\
\hline & Acidobacteria.Acidobacteriia.Acidobacteriales.Acidobacteriaceae & $4.27 * * *$ \\
\hline & Acidobacteria.Acidobacteriia.Acidobacteriales & $4.27 * * *$ \\
\hline & Acidobacteria.Acidobacteriia & $4.27 * * *$ \\
\hline \multirow{5}{*}{$\begin{array}{c}\text { Acer } \\
\text { rubrum }\end{array}$} & Proteobacteria.Alphaproteobacteria.Rhizobiales.Methylocystaceae & $5.13 * * *$ \\
\hline & Proteobacteria.Betaproteobacteria & $4.79 * * *$ \\
\hline & Proteobacteria.Betaproteobacteria.Burkholderiales & $4.79 * * *$ \\
\hline & Proteobacteria.Betaproteobacteria.Burkholderiales.Oxalobacteraceae & $4.77 * * *$ \\
\hline & Proteobacteria.Alphaproteobacteria.Rickettsiales.Rickettsiaceae & $3.81 * * *$ \\
\hline \multirow{5}{*}{$\begin{array}{c}\text { Acer } \\
\text { saccharum }\end{array}$} & Proteobacteria.Alphaproteobacteria.Rhizobiales & $5.18 * * *$ \\
\hline & Bacteroidetes.Cytophagia.Cytophagales.Cytophagaceae.Hymenobacter & $4.48 * * *$ \\
\hline & Proteobacteria.Alphaproteobacteria.Rhizobiales.Beijerinckiaceae & $4.47 * * *$ \\
\hline & Proteobacteria.Alphaproteobacteria.Rhizobiales.Beijerinckiaceae.Beijerinckia & $4.47 * * *$ \\
\hline & Actinobacteria.Actinobacteria.Actinomycetales.Microbacteriaceae & $4.33 * * *$ \\
\hline \multirow{5}{*}{$\begin{array}{c}\text { Betula } \\
\text { papyrifera }\end{array}$} & Proteobacteria.Alphaproteobacteria & $5.39 * * *$ \\
\hline & Proteobacteria & $5.28 * * *$ \\
\hline & Proteobacteria.Alphaproteobacteria.Rhodospirillales & $5.26 * * *$ \\
\hline & Proteobacteria.Alphaproteobacteria.Rhodospirillales.Acetobacteraceae & $5.25 * * *$ \\
\hline & Proteobacteria.Alphaproteobacteria.Rickettsiales & $4.13 * * *$ \\
\hline \multirow{5}{*}{$\begin{array}{l}\text { Picea } \\
\text { glauca }\end{array}$} & Bacteroidetes & $4.97 * * *$ \\
\hline & Bacteroidetes.Cytophagia.Cytophagales & $4.74 * * *$ \\
\hline & Bacteroidetes.Cytophagia & $4.74 * * *$ \\
\hline & Actinobacteria & $4.73 * * *$ \\
\hline & Bacteroidetes.Cytophagia.Cytophagales.Cytophagaceae & $4.73 * * *$ \\
\hline
\end{tabular}

1 